\section{Nuclear Oncology: Pathology and Clinical Applications}

H.W. Strauss, G. Mariani, D. Volterrani, and S. Larson, Eds.

New York, NY: Springer, 2013, 856 pages, $\$ 189$

This book contains 7 parts varying from 1 to 19 chapters in length and totaling 31 chapters. The 6 basic-science chapters cover cancer biology, radiopharmaceuticals, instrumentation, and radiobiology. The clinical chapters cover 18 different cancer types, in addition to including a chapter on pediatric cancers. The latter part of the book is dedicated to radionuclide therapy, guided surgery, the roles of cardiac imaging in cancer care, and adverse-effect monitoring. The last chapter is an illustration of nuclear oncology by case histories and images.

The first 6 chapters are a succinct review of scientific foundations written by leading scientists in nuclear medicine. These chapters are up to date, starting with the hallmarks of cancer, which are necessary for an understanding of nuclear oncology. The clinical chapters are written by expert clinicians and include reviews of epidemiology, tumor classification, imaging, and monitoring of specific tumors. As stated in the title of the book, the emphasis is on clinical applications and the practice of the art. The radionuclide-therapy chapters deal with ${ }^{131}$ I-metaiodobenzylguanidine and bone metastasis targeting. Surprisingly, thyroid cancer therapy is included only in the thyroid cancer chapters, and recent controversies and treatment guidelines are not discussed in detail. The radionuclide-guided surgery chapter on lymphoscintigraphy and parathyroid imaging is aptly written on the current state of the art. This book ends with 21 interesting case studies illustrated by hybrid imaging, mostly with PET/CT, and in one case (case 4) with SPECT/CT. Although the separation of parts and chapters is unusual, with 4 parts (IV, V, VI, and VII) having only a single chapter each, this arrangement does not adversely affect the good flow of information from one part to the next.

In summary, this well-written treatise on nuclear oncology will help nuclear physicians and radiologists who practice nuclear oncology to gain an in-depth understanding of how far this discipline has evolved. The book will also enable scientists who are involved in nuclear oncology research or clinical research to grasp the breadth of the discipline.

\section{Franklin C. Wong}

M.D. Anderson Cancer Center

1515 Holcombe Blvd., Unit 59

Houston, TX 77030

E-mail:fwong@di.mdacc.tmc.edu

Published online Jul. 12, 2013.

DOI: 10.2967/jnumed.113.128769 